\title{
Energy Attacks and Defense Techniques for Wireless Systems
}

\author{
Sheng Wei Jong Hoon Ahnn Miodrag Potkonjak \\ Computer Science Department \\ University of California, Los Angeles (UCLA) \\ Los Angeles, CA 90095 \\ \{shengwei, jhahnn, miodrag\}@cs.ucla.edu
}

\begin{abstract}
This paper addresses the energy attacks towards wireless systems, where energy is the most critical constraint to lifetime and reliability. We for the first time propose a hardwarebased energy attack, namely energy hardware Trojans (HTs), which can be well hidden in the wireless systems and trigger ultra-high energy increases at runtime. Then, we develop a non-destructive HT detection approach to identify the energy attack by remotely sampling the power profiles of the system and characterizing the gate-level temperatures. Our evaluation results on ISCAS benchmarks indicate the effectiveness of the proposed energy attacks and defense techniques.
\end{abstract}

\section{Categories and Subject Descriptors}

K.6.5 [Management of Computing and Information Systems]: Security and Protection-Physical Security

\section{General Terms}

Security

\section{Keywords}

Wireless security, hardware Trojan, leakage energy

\section{INTRODUCTION}

Wireless communication systems, especially mobile devices, have been widely used in a variety of personal and commercial applications, including the traditional wireless phone services and the emerging Internet mobile applications, such as social networking, mobile banking, and multimedia applications. With the ever increasing popularity of these applications, security and integrity of the devices have become a critical concern. Due to the mobile and wireless nature, security primitives for wireless systems are much more challenging and vulnerable than for traditional computer systems.

Permission to make digital or hard copies of all or part of this work for personal or classroom use is granted without fee provided that copies are not made or distributed for profit or commercial advantage and that copies bear this notice and the full citation on the first page. To copy otherwise, to republish, to post on servers or to redistribute to lists, requires prior specific permission and/or a fee.

WiSec'13, April 17-19, 2013, Budapest, Hungary.

Copyright 2013 ACM 978-1-4503-1998-0/13/04 ...\$15.00.
Both research and practical efforts have been directed towards wireless security at various levels, including software applications, communication channels, as well as hardware systems. Among them, many conventional and new security attacks [5][9][18][1][24][35][16] have been well identified, analyzed, and resolved. However, we note that the security towards one of the most crucial and fundamental components in wireless systems, namely energy consumption, has rarely been discussed until now.

It is well known that all wireless devices are energy constrained, especially with the more and more popular uses of mobile devices in computation-intensive applications, such as high definition video streaming and mobile computing. Although huge efforts have been made on energy reduction and optimizations, the systems are vulnerable to energy attacks that intend to cause high energy increase and reduce the lifetime of the system. For example, an adversary may implant a malware that runs in the background of a smart phone and consumes a large amount of battery power. Besides software attacks, energy attacks at the hardware level pose a more severe threat to the wireless systems, because they are much more challenging to be detected and disabled. For example, an untrusted foundry of the cell phone chip or untrusted third party manufacturer of the phone may have hidden hardware Trojans in the hardware that leak additional energy from the phone.

We target on the energy attacks on wireless systems caused by hardware Trojans (HTs), which are malicious modifications to the hardware systems conducted by an untrusted foundry or manufacturer during the manufacturing process [25][29][32][33]. We show that such an energy attack can be implemented by embedding an ultra-small hardware Trojan trigger to the integrated circuit (IC) of the system, which hides in the circuit and results in huge energy increase on the device. In particular, we for the first time develop and demonstrate two types of hardware energy attacks by manipulating the input vectors and biasing the power supply, respectively. We argue that such energy attacks are extremely difficult to detect. On one hand, the attacker tends to hide the HT trigger in the target circuit by either sizing it ultrasmall or by activating it only when an extremely rare event occurs. On the other hand, the leakage energy by itself has a high dependence on the environment temperatures and, therefore, the attacker could attribute any malicious energy attack to the variations of environment temperatures. It is significantly difficult for the detection process to distinguish between the two cases. 
Based on the investigation of the powerful energy attacks, we develop defense approaches for energy attacks by leveraging power profiling and temperature characterizations. The idea is to sample the total leakage power consumption of the wireless system and characterize the gate-level temperature profile by assuming that there is no energy attack. In the case where there is indeed malicious energy attack, the characterized temperature profile will not meet the normal spatial and temporal thermal distributions on an IC. We identify the possible discrepancy in temperatures quantitatively by defining a hardware Trojan indicator concerning the spatial inconsistency of the gate-level temperatures. Note that our energy HT detection approach requires no instrumentation to the wireless system, which can be conducted remotely without having direct physical access to the target device.

Figure 1 shows the overall flow of our approaches for energy attack and defense, which spreads over the wireless system manufacturing, post-silicon testing, and real time (in-field) operation. Firstly, we investigate on the energy HT attack that can be conducted by an adversary during the manufacturing process, where the design objective is to maximize the potential damage (i.e., the leakage energy consumption) and minimize the probability of being detected (i.e., placing the trigger of the HT at a rarely switching or low leakage location, as well as enabling the sequentialbased activation event). Note that we develop two attack techniques, namely input vector control and forward body biasing, which significantly increase the leakage energy in the sleep mode and operation mode of the wireless system, respectively. Secondly, we employ a gate-level temperature characterization method to monitor and identify abnormal leakage energy variations at the runtime. Our approach characterizes the gate-level temperature profiles via energy profiling and thus distinguishes the abnormal energy hike due to security attacks and the normal energy variations due to environmental factors.

To the best of our knowledge, this is the first complete analysis and implementation of hardware-based energy attacks and defense techniques in wireless systems. In summary, our technical contributions include:

- Attack: a powerful and well hidden hardware energy attack to wireless systems by embedding and hiding hardware Trojan components in the target circuit;

- Defense: a temperature-aware power profiling approach to detect energy hardware Trojans in the wireless systems without embedding any additional hardware to the system.

The remainder of this paper is organized as follows. In Section 2, we summarize the existing research work regarding hardware Trojan detection, thermal-aware IC design, and adaptive body bias. Section 3 introduces the leakage energy model and the gate-level characterization approach that we employ in this work. In Section 4, we introduce the design of the high leakage HT in both the sleep mode and the system operation mode; Section 5 discusses our detection techniques against the high energy attack, which identifies the presence of attacks via gate-level temperature characterization. We show our experimental results in Section 6 and conclude the paper in Section 7.

\section{RELATED WORK}

In this section, we summarize the existing research efforts in the areas of hardware Trojan detection, thermal-aware IC design, and adaptive body bias techniques, with the emphasis of our new contributions compared to the previous approaches and techniques.

\subsection{Hardware Trojan Detection}

Hardware Trojans (HTs) [25][29][32][33][13][26] are malicious modifications to integrated circuits (ICs) that are possibly conducted by untrusted CAD tools at design time, or untrusted foundries during manufacture. With the ever increasing trend of IC outsourcing, the security and reliability concerns caused by hardware Trojans have drawn a great deal of attention in the IC design and security community. Furthermore, since ICs are fundamental building blocks of embedded systems, where the security primitives are much more challenging and vulnerable than traditional hardware systems, it is crucial to evaluate and detect the HTs.

Tehranipoor et al. [25] provided a comprehensive survey of HT detection. Recently, more and more efforts have been made in developing side-channel (e.g., power and delay) based detection techniques [2][19][12][15][29][31][28].

The existing HT detection efforts targeted only on HTs that cause direct security attacks, such as implanting a backdoor in the circuit and extracting confidential information from the system at runtime. However, we note that the security toward one of the most crucial and fundamental components in embedded systems, namely energy consumption, has rarely been discussed until now. Our energy HT detection approach is new compared to the existing HT detection techniques. We for the first time analyze and address hardware Trojans of energy attacks towards wireless systems, which has not been discussed in the current literature.

\subsection{Thermal-aware IC Design and Analysis}

Thermal effect has become a crucial factor being considered in IC design and manufacturing because of the interdependency between IC properties (i.e. delay and leakage power) and temperature. Recently, many research efforts have been made on thermal-aware leakage model and leakage reduction techniques. Li et al. [20] developed an architectural model for subthreshold and gate leakage that explicitly defines the relationship between leakage power and temperature. It shows that the subthreshold leakage currents are exponentially dependent on temperature and voltage. Besides leakage power, Liao et al. [21] showed that the performance of IC also depends on temperature.

Temperature monitoring of IC systems has drawn a great deal of attention in the IC design and manufacturing community. Finite element analysis (FEA) [38] and resistor networks [7] approaches employ heat transfer model for semiconduct materials and calculate the IC temperature profiles at design time. Power blurring technique [14] reduces the computation time of FEA by using matrix convolution. However, these techniques are designed for temperature calculation and prediction at the design time, which is not resilient to the process variation and cannot be used for runtime temperature monitoring. At runtime, thermal sensorbased approaches [27] have been proposed to measure the temperatures in the real time. However, these approaches introduce a high overhead and instrumentation to the target circuit. 


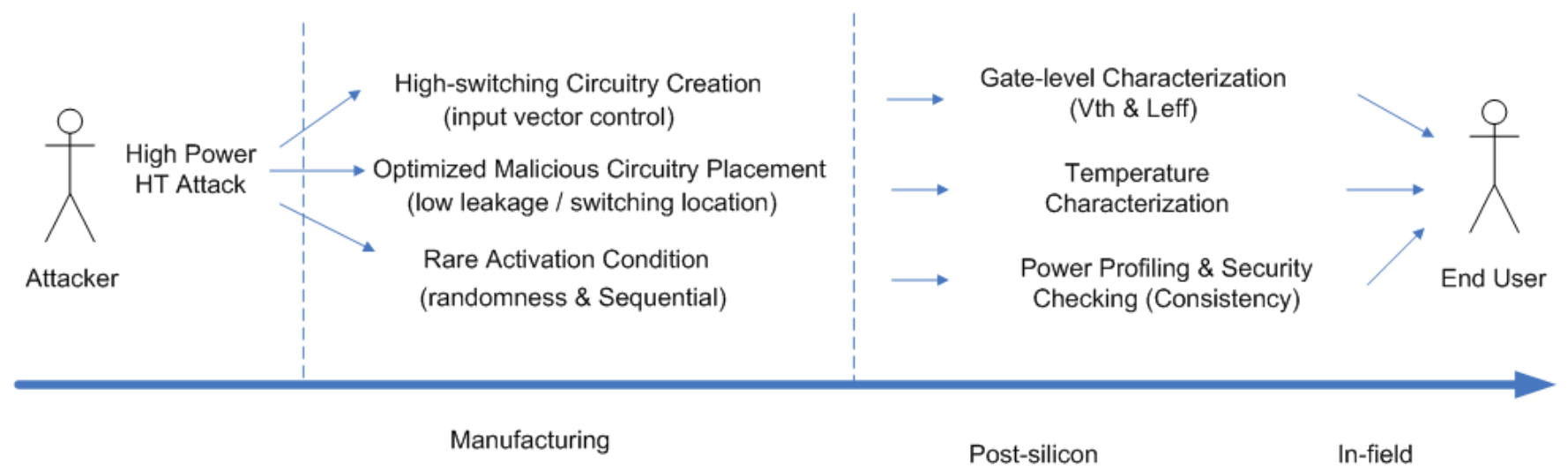

Figure 1: Overall flow of high power attack and defense.

Compared to the existing approach, our gate-level temperature profiling technique is new in the following aspects. First, our approach is non-destructive, as we do not require any additional hardware to be added in the target circuit, which minimizes the overhead; Second, we take into consideration of the process variation and measurement error, which enables us to monitor the real time temperatures for remote wireless systems. For example, our technique can also be used to facilitate remote hardware identification or watermarking [11][17][39].

\subsection{Adaptive Body Bias}

Adaptive body bias (ABB) has been widely adopted as an efficient post-silicon approach in the research efforts of leakage energy reduction and performance optimization [23][10][6]. For example, Nabaa et al. [23] proposed the use of ABB through the use of the new FPGA architecture that includes an additional characterizer circuit to reduce the leakage energy by 3 times. Gregg et al. [10] proposed using ABB to compensate for the process variation and improve delay and leakage. Chen et al. [6] compare the effectiveness of adaptive supply voltage (ASV) and ABB. Furthermore, similar with the pre-silicon dual $V_{t h}$ approach, researchers have proposed multiple body bias values in the target circuit, each drives a subset of the gates. For example, Xu et al. [36] cluster the gates at a finer-grained level and apply multiple $\mathrm{ABB}$ values to control the leakage energy consumption.

Compared to the existing work, We investigate on an unconventional use of the ABB techniques, possibly by an adversary, to increase the leakage energy consumption of wireless systems. Instead of compensating for the process variation and reducing leakage energy consumption, an attacker may apply forward bias voltages to increase the leakage energy exponentially.

\section{PRELIMINARIES}

In this section, we summarize the preliminaries and key observations that serve as the foundation of our proposed energy attack and defense techniques, including the energy model we employ for evaluating the leakage energy, as well as the gate-level characterization scheme that recovers the gate-level physical properties from global leakage energy measurements.

\subsection{Energy Model}

We consider leakage energy and switching energy that are major sources of energy consumption during IC operations. The leakage energy is dependent on the IC physical properties such as effective channel length $L_{\text {eff }}$ and threshold voltage $V_{t h}$. Equation (1) is the gate-level leakage energy model [22], where $W$ is gate width, $L$ is gate length, $V_{t h}$ is threshold voltage, and $T$ is the temperature. The rest of the parameters are considered as constants and are discussed in details in [22].

$$
P_{\text {leakage }}=2 \cdot n \cdot \mu \cdot C_{o x} \cdot \frac{W}{L} \cdot(k T / q)^{2} \cdot D \cdot V_{d d} \cdot e^{\frac{\sigma \cdot V_{d d}-V_{t h}}{n \cdot(k T / q)}}
$$

Equation (1) indicates that the leakage energy of logic gate depends on the temperature in a non-linear manner. Therefore, any temperature changes in the environment, or due to the switching of the gates in the circuit, will have impact on the energy consumption of the IC system.

The gate-level switching energy model [22] is described by Equation (2), where the switching energy is dependent on gate width $W$, gate length $L$, and supply voltage $V_{d d}$. where $\alpha$ is the switching probability.

$$
P_{\text {switching }}=\alpha \cdot C_{o x} \cdot W \cdot L \cdot V_{d d}^{2}
$$

\subsection{Gate-level Characterization}

In gate-level characterization (GLC), we recover the gatelevel IC properties from global side-channel measurements under the application of various input vectors [29][30][34]. For example, when $J$ input vectors have been applied on a target circuit with $K$ gates, the gate-level leakage energy values can be solved using the following linear program (LP):

$$
\begin{array}{cl}
\text { Objective : } & \min _{1 \leq j \leq J} \mathcal{F}\left(\operatorname{err}_{j}\right) \\
\text { Constraints : } & \sum_{k=1}^{K} E_{j k}=\tilde{E}_{j}+e r r_{j} \\
& j=1, \ldots, J
\end{array}
$$

where $E_{j k}$ is the leakage energy of gate $k(k=1, \ldots, K)$ when input vector $j(j=1, \ldots, J)$ is applied; $\tilde{E}_{j}$ is the measured total leakage energy when the input vector $j$ is applied; $e r r_{j}$ is the measurement error; $\mathcal{F}$ is a metric for quantifying the measurement errors, such as $l_{1}$ or $l_{2}$ norm. In this LP formulation, $E_{j k}$ can be expressed as a product 
of its constant nominal value $E_{n o m, j k}$ and a scaling factor (due to PV) $\delta_{k}$, i.e., $E_{j k}=\delta_{k} E_{n o m, j k}$. By solving the LP with $\delta_{k}$ as the variables, we can obtain the value of $E_{j k}$ for each gate $k(k=1, \ldots, K)$. Furthermore, by following the energy models (i.e., Equations (1) and (2)), we can formulate a system of nonlinear equations and solve for the physicallevel properties (i.e., threshold voltage and effective channel length) of each individual gate.

\section{HIGH LEAKAGE ENERGY ATTACK}

In this section, we design and analyze energy attacks on wireless systems via malicious hardware modification during the manufacturing process (i.e., energy hardware Trojans). We analyze two major components of the hardware Trojans, namely (1) action, which indicates what attacks the HT could impose and how the attacks are implemented; and (2) trigger, which indicates the activation condition that would trigger the energy attack. By analyzing these two components, we aim to quantify the energy increase caused by the attacks as well as the probability of activation, which are quantitative indicators of the effectiveness of attacks and the difficult level for detection, respectively. An attacker would tend to maximize the action while reducing the trigger probability to impose damaging and well hidden energy attacks.

\subsection{Sleep-mode Energy Attack: Input Vector Manipulation}

For most of the wireless systems and applications, the circuit of the system would stay in the sleep mode for a large portion of the time. For example, in the case of a cell phone, the major components are only exercised once it is in a voice call. Similarly, in a wireless sensor network, the communication circuitry only operates during the data collection process. Consequently, the input vectors that are being applied during the IC sleep mode becomes crucial with regard to the leakage energy consumption, because of the fact that the leakage energy of a logic gate highly depends on the input vectors [37]. For example, as shown in Figure 2, the leakage energy of a NAND gate can vary up to 12 times with different input vectors. Although this phenomenon can provide us with an opportunity for leakage energy reduction, it is more easily leveraged by an attacker for energy attack.

\begin{tabular}{|c|c|}
\hline Input & Leakage Current \\
\hline 0 & $100.3 \mathrm{nA}$ \\
\hline 1 & $227.2 \mathrm{nA}$ \\
\hline
\end{tabular}

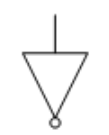

\begin{tabular}{|c|c|}
\hline Input Vector & Leakage Current \\
\hline 00 & $37.84 \mathrm{nA}$ \\
\hline 01 & $95.17 \mathrm{nA}$ \\
\hline 10 & $100.3 \mathrm{nA}$ \\
\hline 11 & $454.5 \mathrm{nA}$ \\
\hline
\end{tabular}

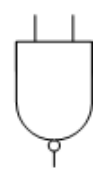

Figure 2: Leakage current of an inverter and a NAND gate under different input vectors [37].

Figure 3 shows a motivational example of energy attack using input vector manipulation. In this case, the HT component applies an input vector that sets the maximum number of gates in the high energy state, which results in the highest energy consumption (i.e., 2.96 times of the minimum energy consumption). If this situation continues over time without being identified by the user or tester of the system, it will cause several times more energy consumption, which is considered significant in a power hungry system.

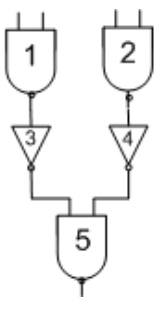

Attack Input Vector: 1111

Leakage $=3 \times 454.5+2 \times 100.3=1564.1 \mathrm{nA}$

Optimal Input Vector: 0000

Leakage $=3 \times 37.84+2 \times 227.2=530.08 \mathrm{nA}$

Figure 3: Example of energy attack via input vector manipulation. The energy consumption caused by the attack input vector is 2.96 times compared to the optimal input vector.

\subsection{Operation-mode Energy Attack: Forward Adaptive Body Bias}

During IC operation, the supply voltage plays an important role to the total leakage power consumption. According to Equation (1), the leakage energy of a transistor increases exponentially with the increase of supply voltage. We argue that this phenomenon can be leveraged for powerful energy attacks, since an exponential energy increase could cause huge impact to the wireless system and thus becomes the best interest of an attacker. In particular, we argue that an attacker could possibly apply a forward adaptive body bias (FBB, or forward ABB) voltage that, instead of compensating for the process variation as in the normal use of body biasing techniques [6][10][36], would increase the energy exponentially in the circuit under attack.

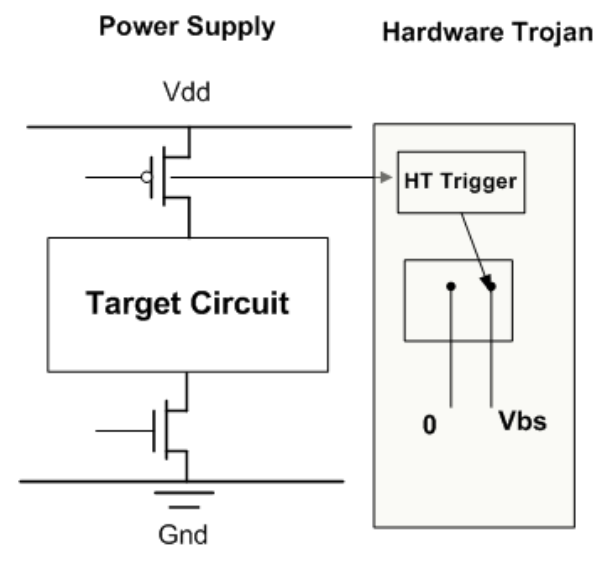

Figure 4: Example of energy attack using forward body biasing.

With this consideration, we implement a sample malicious circuitry that triggers the FBB-based energy attack, as shown in Figure 4. The shaded part of the circuit is the malicious component, or HT, embedded by an adversary. During the operation mode, once triggered, the HT can select and apply a forward body bias voltage, which increases 
the supply voltage and maximizes the leakage energy without compromising the functionality of the system.

\subsection{HT Triggers: Rare Activation of Attacks}

In the previous two subsections, we have shown that HTbased energy attack could cause huge energy increase, either linearly in the sleep mode or exponentially in the operation mode. However, the attacks would not take effective unless the triggers are well hidden from the common detection approaches. In this subsection, we discuss in details how an attacker could design the trigger such that the resulting HT attack has a low probability to be detected by the HT detection attempts. The intuitions of hiding the HT trigger, from the attacker's perspective, include the following: (1) hide the HT trigger in the circuit both physically and in terms of their observable properties, such as delay and power; and (2) minimize the activation probability of the HT.

\subsubsection{Hiding the HT trigger}

In order to bypass the most commonly used side channelbased detection approaches, an attacker aims to place the HT trigger in such a way that it is non-observable via the commonly considered side channels, including delay, leakage power, and switching power. In order to achieve this goal, we add only one single gate in the target circuit that serves as the trigger. With this single gate HT trigger, we ensure that any resulting delay or power variation is minimum to increase the difficulty level for detection. Furthermore, to further complicate the side channel-based detection approaches, we place the single gate HT trigger at a circuit location where the delay and power are non-observable or difficult to measure. For example, in Figure 5(a), the delay of the HT trigger is non-observable due to the parallel reconvergent paths. One can measure the delay between the two endpoints $x$ and $y$. However, it is not possible to determine whether the measured delay is for path 1 or path 2 and, therefore, the HT can be hidden under the delay measurements.

\subsubsection{Minimizing the activation probability}

Based on the HT trigger placement that is difficult to detect, we further reduce its activation probability to bypass the security checks that are based on generated test vectors, such as automatic test pattern generation (ATPG) [2]. The idea is to set the activation condition in such a way that it is only known to the attacker and very rarely triggered during a normal IC operation or test. We achieve this goal by using two approaches. Firstly, we select the fan-in gates from the target circuit in such a way that the HT trigger is rarely switched, as shown in an example in Figure 5(b). The activation probability of the NAND HT trigger is $1 / 2^{n}$, where $n$ is the number of inputs that can be customized by the attacker to balance the trade-off between the size of the HT trigger and the activation probability. Secondly, we leverage sequential elements (i.e., flip-flops) that create temporal-based activation conditions in a finite state machine (FSM). In this way, the activation probability of the HT trigger can be further reduced exponentially based on the results from the first approach. For example, as shown in Figure 5(c), the 5-state FSM serves as the activation condition, which triggers the HT only when all 5 states are satisfied in 5 consecutive clock cycles. As a result, the ac- tivation probability is $\prod_{i=1}^{m} P_{i}$, where $P_{i}$ is the activation probability of the vector in state $i$, and $m$ is the number of states.

\section{TEMPERATURE-AWARE CONSISTENCY- BASED HT DETECTION}

In this section, we discuss our detection approach to identify the HT-based energy attacks. In order to exclude the possible impact of temperature in energy increase, which is likely to be claimed by the attacker, we conduct gatelevel temperature characterization to recover the temperature profile of the circuit. Then, we calculate the value of our defined HT indicator, which is the spatial inconsistency of temperatures among physically adjacent gates, to determine the presence of energy attack.

\subsection{Energy Paradox}

The most straightforward detection approach towards the energy attack is by sampling the energy profiles of the operating wireless systems on a regular basis and observe the abnormal energy increase. Although remote sampling and data collection is a common practice for wireless system performance or status monitoring, the collected power profile is not an effective indicator for energy attacks, due to the following energy paradox, which can be leveraged by energy attackers:

Energy Paradox. Due to the exponential dependence of leakage energy on temperatures, as indicated in Equation (1), it is not certain to the system user whether the energy increase is caused by normal temperature variations or malicious energy attacks. As a matter of fact, it is common that the target wireless system, such as a wireless sensor network, is deployed in a hazardous environment where the temperature varies in an unknown pattern. In this case, the power profiling approach by itself is not sufficient to reach a conclusive judgment of whether any energy attack exists or not.

\subsection{Gate-level Temperature Characterization}

In order to address the energy paradox and obtain accurate energy HT detection results, in the case of a high energy profile, we must measure or characterize the temperature of the target IC to either exclude its impact or report that the energy increase is due to temperature. Several approaches have been proposed in monitoring the temperatures of IC systems, such as FEA [38], power blurring [14], and sensors [27]. However, the FEA and power blurring approaches work at the IC design stage without the taking account of the impact of process variation and are not resilient for post-silicon attacks. The sensors-based approaches provide real-time measurements of temperatures, but they require additional sensor circuitry in the target IC, which greatly increases the complexity and cost of the system.

We develop a non-destructive gate-level temperature characterization approach using power profiling, which does not require additional hardware circuitry being added to the target IC. The approach is based on the physical-level GLC concerning threshold voltage $\left(V_{t h}\right)$ and effective channel length $\left(L_{e f f}\right)$. We show the flow of temperature characterization in Pseudocode 1. Firstly, before the release and deployment of the wireless system, we characterize the gate-level $V_{t h}$ and 


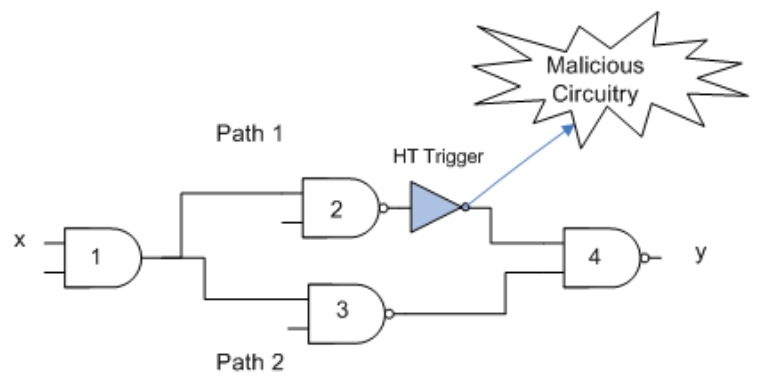

(a)

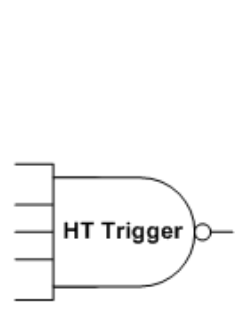

(b)

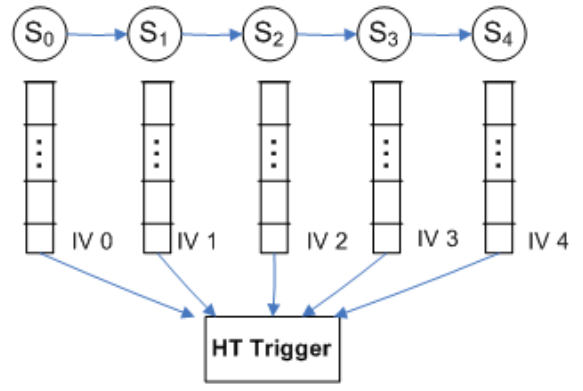

(c)

Figure 5: Example of hiding the HT triggers: (a) a HT trigger embedded in the reconvergent paths where delay is non-observable; (b) a HT trigger activated by a 5-state finite state machine, which reduces the activation probability exponentially.

$L_{e f f}$ at room temperature, where we assume the temperature $T$ in Equation (1) as a constant value. Then, after the system has been deployed and in operation, we take $M$ power measurements and characterize the temperature $\left(T_{i}\right)$ of each gate using Equation (1) based on the $V_{t h}$ and $L_{\text {eff }}$ that are already known. Finally we conduct online security checking to determine the presence of energy attack using the HT indicator, as defined and discussed in the next subsection.

Pseudocode 1 Gate-level temperature characterization via power profiling.
1: Post-silicon:
2: Gate-level characterization to solve $V_{t h}, L_{\text {eff }}$ of each gate at room temperature following Equations (1) and (2);
3: Runtime:
4: Take $M$ power measurements via sampling;
5: for all Gates $g_{i}$ in the circuit do
6: $\quad$ Solve for temperature $T_{i}$ following Equations (1);
7: end for
8: Security Check:
9: Conduct security check on temperature $T_{i}$ over all gates;

\subsection{HT Indicator}

The problem we face in inspecting the characterized temperature profile for HT detection is that the normal temperature profile, or the "golden model", is not available in the case of wireless systems. It is because the system is often deployed in unknown environments (e.g., wireless sensor network) or has a mobile nature and a high probability of environmental changes (e.g., smart phones). Therefore, the online temperature security inspection cannot be done via simple comparisons.

We solve the problem by defining a HT indicator that represents the temperature inconsistency over gates that are adjacent to each other in the target IC. Our intuition is that the heat transfer process would create spatial correlations in the temperatures of gates that are physically close to each other. Therefore, if we ever observe that there is an abnormally large deviation between the temperatures of two or more adjacent gates, it is an indicator that the energy increase is not likely caused by temperature changes but by malicious energy attacks. This is based on the assumption that it is computationally impossible for an attacker to emulate the heat transfer model and impose energy attacks following exactly the same pattern.

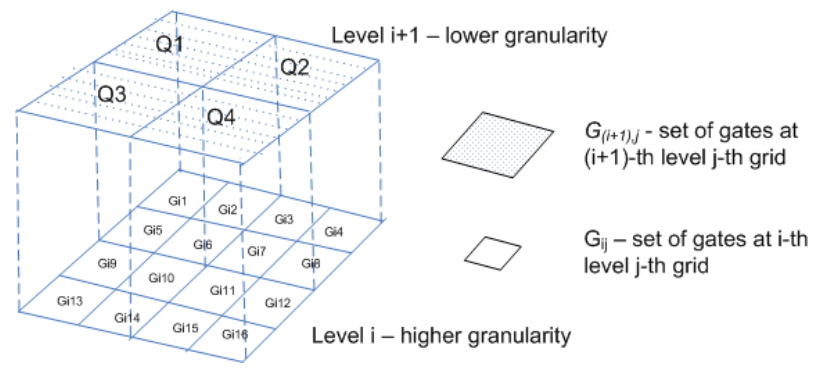

Figure 6: Principal component analysis (PCA) model to define the hardware Trojan indicator.

We define the HT indicator using the principal component analysis (PCA) models [8] that were originally used for modeling spatial correlations in IC process variations. As shown in Figure 6, we group the gates into multiple grids at different levels in order to capture the inconsistency of the temperatures between various boundaries of adjacent gates. At each specific level, we define the HT indicator as the average standard deviation, over all grids, of temperatures among all gates within each grid. In particular, at the $i$-th level, the HT indicator $H_{i}$ can be calculated as the following:

$$
H_{i}=\left(\sum_{j=1}^{N_{i}} \operatorname{stddev}\left(G_{i j}\right)\right) / N_{i}
$$

where $N_{i}$ is the number of grids at level $i$, and $G_{i j}$ is the set of gates in the $j$-th grid at level $i$. We use $H_{i}$ to evaluate the temperature deviation over adjacent gates at different granularities. Depending on the sizes and physical properties of the circuit under test, different levels of $H_{i}$ plays different roles in the final evaluation of the temperature deviations. Therefore, we define the following weighted function for the overall HT indicator: 


$$
H=\left(\sum_{i=1}^{L} w_{i} H_{i}\right) / L
$$

where $L$ is the number of levels that we divide using the PCA model, and $w_{i}$ is the weight factor at level $i$ concerning the physical properties of the circuit.

\section{EXPERIMENTAL RESULTS}

We evaluate our temperature-aware energy HT detection approach on a set of ISCAS benchmarks that are widely used in the IC design and hardware security community [4][3]. In this section, we discuss in details our evaluation results in the energy HT attack and defense.

\subsection{Effectiveness of Energy HT Attack}

We evaluate the effectiveness of energy HT attack from two aspects that are essential for a hardware Trojan: (1) HT action, i.e., how much energy increase the energy HT causes to the circuit under attack; and (2) HT trigger, i.e., the probability of activation of the HTs, which indicates how well they can be hidden from common detection approaches.

\subsubsection{Energy Increase}

We evaluate the energy increase caused by forward ABBbased energy attack by inserting a HT trigger that selects ABB voltages up to $1.0 \mathrm{~V}$. Figure 7 shows the energy increase due to the attack on a set of ISCAS benchmarks. We observe that the energy consumption grows exponentially with the linear increase of the $\mathrm{ABB}$ voltage, creating huge impacts on the circuit under attack.

\subsubsection{Activation Probabilities of the Energy HTs}

We further evaluate the activation probabilities of the HT triggers in order to quantify the difficulty levels for detecting these energy attacks. Table 1 shows our simulation results on a set of ISCAS benchmarks. We evaluate two cases with the number of fan-in gates for the HT trigger selected from the target circuit varying between 5 and 10 . In each case, we employ a 5-state finite state machine that randomly generates 5 sequential activation input vectors that serve as the activation condition of the HT trigger. Our results show that the activation probabilities are extremely low and decrease exponentially with the increase of fain-in signals and the number of states in the finite state machine. The probabilities are low enough to create challenging attacks that are computationally infeasible to be covered by any existing functional test detection schemes.

\subsection{Effectiveness of Temperature-aware HT De- tection}

We evaluate the effectiveness of our temperature-aware HT detection approach from two aspects. Firstly, we evaluate the accuracy of the gate-level temperature characterization, which is an indicator of how accurate we can capture the abnormal temperature variations. Then, we evaluate the HT detection approach by comparing the HT indicator values in two cases where HTs are present and where there are no HTs, in order to determine the false positives and false negatives in HT detection.

\subsubsection{Accuracy of Gate-level Temperature Charac- terization}

We evaluate the accuracy of the gate-level temperature characterization by comparing the characterized temperatures and the actual temperatures and quantifying the average characterization errors. Figure 8 shows the distribution of the characterization errors for each gate in a set of ISCAS benchmarks. The results indicate that characterization errors of all gates are controlled within the $2 \%$ mark except for very few outliers gates. Also, the accuracy does not decrease with the increase of the circuit sizing, indicating the scalability of our detection approach.

\subsubsection{Effectiveness of Detection Using HT Indicator}

In order to evaluate the effectiveness of HT detection, we characterize the gate-level temperature profiles in two cases where there are no energy attacks (i.e., HT-free) and where there are forward ABB-based energy HTs embedded and triggered in the target circuit (i.e., HT-present). For each case, we calculate the value of the HT indicator as defined in Section 5.3 to observe the difference between the two cases. In our simulation, we use 3 level of grids in the PCA model $(\mathrm{L}=3)$ and use evenly assigned weight factors for the $w_{i}$ (i.e., $\left.w_{i}=1 / 3, i=1,2,3\right)$ in calculating $H$. Figure 9 shows our evaluation results of HT indicator $H$ in both the HT-present and HT-free cases. There is an obvious and large difference between the HT indicators in the two cases. In the HTpresent case, the HT indicator is significantly larger than that of the HT-free case. Therefore, the HT indicator is a good metric for differentiating the two cases, which provides us with zero false positives and zero false negatives in the detection of energy HTs.

\section{CONCLUSION}

We developed the first hardware Trojan-based energy attack towards wireless systems using input vector manipulation and forward body biasing. To complicate the detection process, we hide the trigger of the energy hardware Trojan by embedding it in unobservable paths and minimizing its activation probability. Then, as defense, we developed a temperature-aware gate level characterization approach that distinguishes between the energy increase caused by normal temperature variations and due to malicious attacks. Our simulation results on ISCAS benchmarks verified the effectiveness of the energy attack and defense approaches. To the best of our knowledge, this is the first attempt to analyze and address hardware Trojan-based energy attacks in wireless systems.

\section{ACKNOWLEDGEMENTS}

This work was supported in part by the NSF under Award CNS-0958369, Award CNS-1059435, and Award CCF-0926127, and in part by the Air Force Award FA8750-12-2-0014.

\section{REFERENCES}

[1] B. Awerbuch, D. Holmer, C. Nita-Rotaru, and H. Rubens. An on-demand secure routing protocol resilient to byzantine failures. In $A C M$ workshop on Wireless security (WiSe), pages 21-30, 2002.

[2] M. Banga and M. Hsiao. A region based approach for the identification of hardware Trojans. In IEEE 

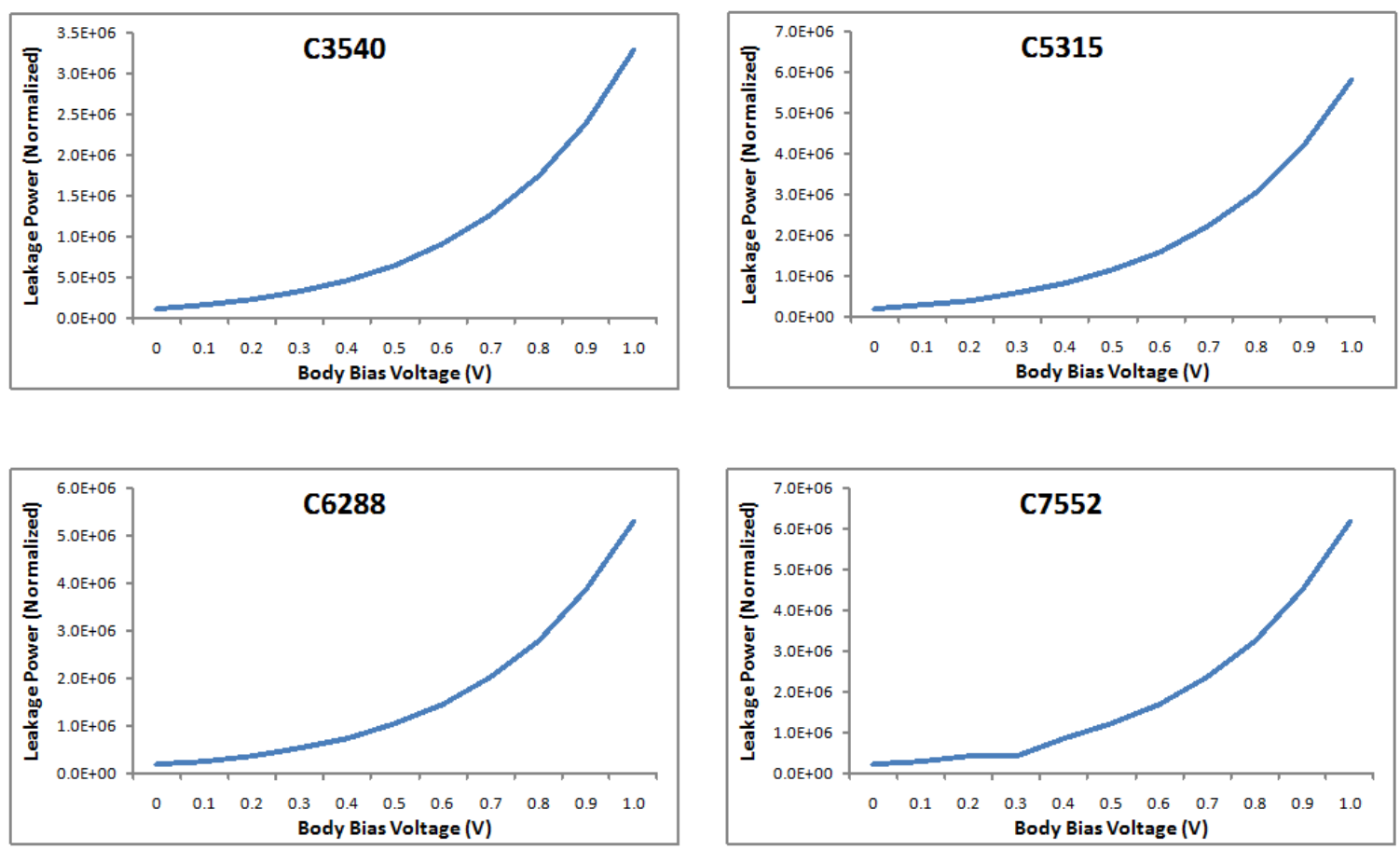

Figure 7: Leakage power increase due to forward ABB attack.

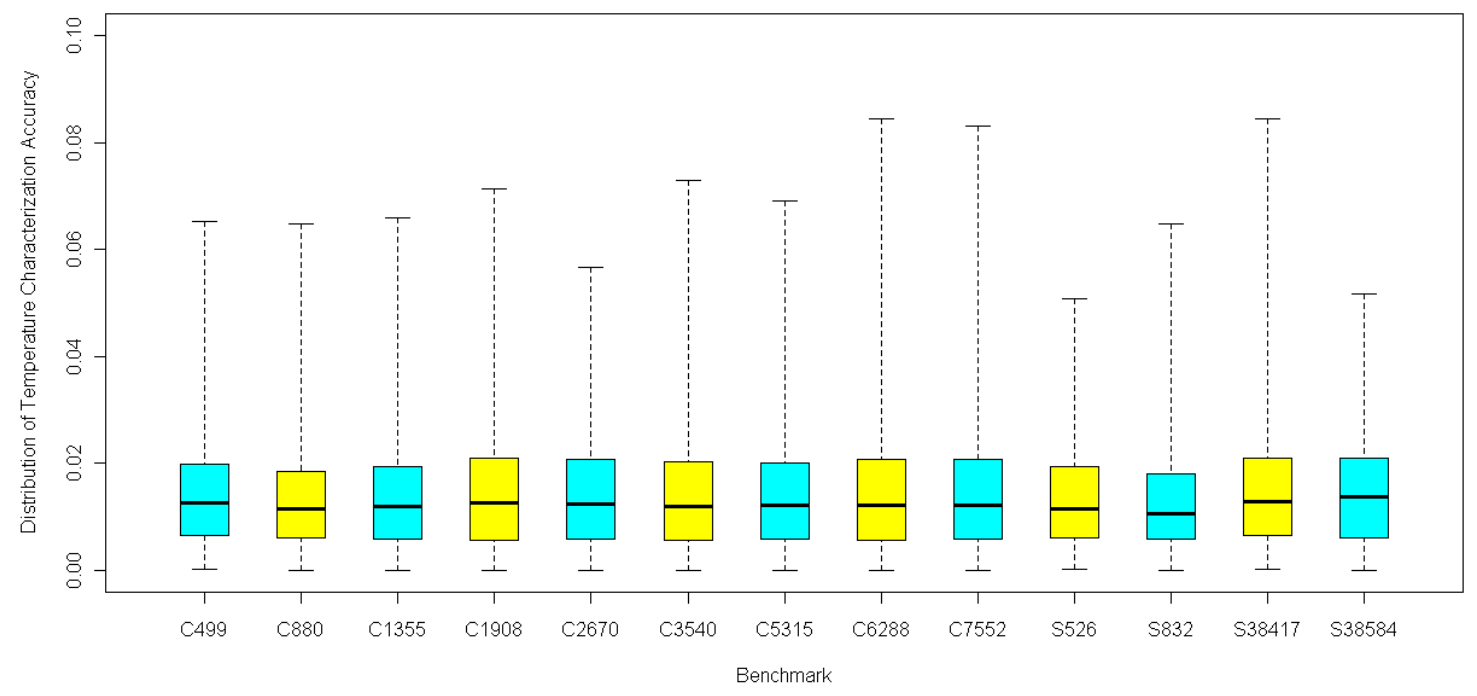

Figure 8: Accuracy of temperature characterization. 
Table 1: Activation probability of hardware Trojans using low-switching and sequential triggers.

\begin{tabular}{|c|c|c|c|c|c|}
\hline Benchmark & \# Gates & \# inputs & \# outputs & Probability of Activation (5-trigger) & Probability of Activation (10-trigger) \\
\hline C432 & 160 & 36 & 7 & $4.8 \mathrm{E}-17$ & $2.4 \mathrm{E}-43$ \\
\hline C499 & 202 & 41 & 32 & $8.9 \mathrm{E}-24$ & $2.0 \mathrm{E}-39$ \\
\hline C880 & 383 & 60 & 26 & $2.9 \mathrm{E}-16$ & $1.1 \mathrm{E}-35$ \\
\hline C1355 & 546 & 41 & 32 & $2.1 \mathrm{E}-37$ & $5.9 \mathrm{E}-44$ \\
\hline C1908 & 880 & 33 & 25 & $3.8 \mathrm{E}-26$ & $4.8 \mathrm{E}-43$ \\
\hline C3540 & 1669 & 50 & 22 & $2.3 \mathrm{E}-23$ & $2.8 \mathrm{E}-46$ \\
\hline C5315 & 2307 & 178 & 123 & $9.5 \mathrm{E}-27$ & $7.0 \mathrm{E}-49$ \\
\hline C7552 & 3512 & 207 & 108 & $7.6 \mathrm{E}-27$ & $2.3 \mathrm{E}-47$ \\
\hline
\end{tabular}

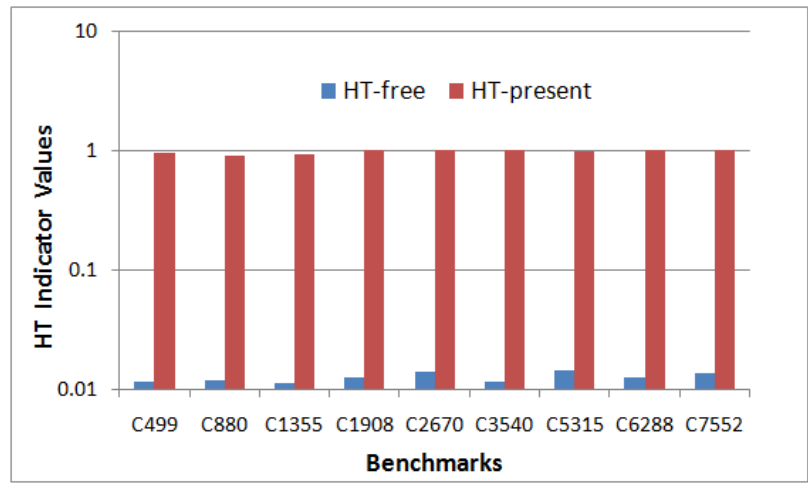

Figure 9: Energy HT detection results: HT indicator values in HT-free and HT-present cases.

International Workshop on Hardware-Oriented Security and Trust (HOST), pages 40-47, 2008.

[3] F. Brglez, D. Bryan, and K. Kozminski.

Combinational profiles of sequential benchmark circuits. In IEEE International Symposium on Circuits and Systems (ISCAS), pages 1929-1934, 1989.

[4] F. Brglez and H. Fujiwara. A neutral netlist of 10 combinational benchmark circuits and a target translator in FORTRAN. In IEEE International Symposium on Circuits and Systems (ISCAS), pages 677-692, 1985.

[5] S. Capkun, L. Buttyan, and J. Hubaux. Self-organized public-key management for mobile ad hoc networks. IEEE Transactions on Mobile Computing, 2(1):52-64, 2003.

[6] T. Chen and S. Naffziger. Comparison of adaptive body bias (ABB) and adaptive supply voltage (ASV) for improving delay and leakage under the presence of process variation. IEEE Transactions on Very Large Scale Integration (VLSI) Systems,, 11(5):888-899, 2003.

[7] Y. Cheng, C. Tsai, C. Teng, and S. Kang.
Temperature-driven cell placement. In Electrothermal Analysis of VLSI Systems, pages 157-179, 2000.

[8] B. Cline, K. Chopra, D. Blaauw, and Y. Cao. Analysis and modeling of $C D$ variation for statistical static timing. In IEEE/ACM International Conference on Computer-Aided Design (ICCAD), pages 60-66, 2006.

[9] L. Eschenauer and V. Gligor. A key-management scheme for distributed sensor networks. In $A C M$ Conference on Computer and Communications Security (CCS), pages 41-47, 2002.

[10] J. Gregg and T. Chen. Post silicon power/performance optimization in the presence of process variations using individual well-adaptive body biasing. IEEE Transactions on Very Large Scale Integration (VLSI) Systems, 15(3):366-376, 2007.

[11] I. Hong and M. Potkonjak. Behavioral synthesis techniques for intellectual property protection. In Design Automation Conference (DAC), pages 849-854, 1999.

[12] Y. Jin and Y. Makris. Hardware Trojan detection using path delay fingerprint. In IEEE International Workshop on Hardware-Oriented Security and Trust (HOST), pages 51-57, 2008.

[13] R. Karri, J. Rajendran, K. Rosenfeld, and M. Tehranipoor. Trustworthy hardware: Identifying and classifying hardware Trojans. IEEE Computer Magazine, 43(10):39-46, 2010.

[14] T. Kemper, Y. Zhang, Z. Bian, and A. Shakouri. Ultrafast temperature profile calculation in IC chips. In International Workshop on Thermal investigations of ICs, pages 1-5, 2006.

[15] F. Koushanfar and A. Mirhoseini. A unified framework for multimodal submodular integrated circuits Trojan detection. IEEE Transactions on Information Forensics and Security, 6(1):162-174, 2011.

[16] F. Koushanfar and M. Potkonjak. CAD-based security, cryptography, and digital rights management. In Design Automation Conference (DAC), pages 268 $-269,2007$.

[17] J. Lach, W. Mangione-Smith, and M. Potkonjak. 
FPGA fingerprinting techniques for protecting intellectual property. In Custom Integrated Circuits Conference, pages 299-302, 1998.

[18] L. Lazos and R. Poovendran. SeRLoc: secure range-independent localization for wireless sensor networks. In ACM workshop on Wireless security (WiSe), pages 21-30, 2004.

[19] J. Li and J. Lach. At-speed delay characterization for IC authentication and Trojan horse detection. In IEEE International Workshop on Hardware-Oriented Security and Trust (HOST), pages 8-14, 2008.

[20] Y. Li, D. Parikh, Y. Zhang, K. Sankaranarayanan, M. Stan, and K. Skadron. State-preserving vs. non-state-preserving leakage control in caches. In Design Automation and Test in Europe (DATE), pages 22-29, 2004.

[21] W. Liao, L. He, and K. Lepak. Temperature and supply voltage aware performance and power modeling at microarchitecture level. IEEE Transactions on Computer-Aided Design of Integrated Circuits and Systems, 24(7):1042-1053, 2005.

[22] D. Markovic, C. Wang, L. Alarcon, T. Liu, and J. Rabaey. Ultralow-power design in near-threshold region. Proceedings of the IEEE, 98(2):237-252, 2010.

[23] G. Nabaa, N. Azizi, and F. Najm. An adaptive FPGA architecture with process variation compensation and reduced leakage. In Design Automation Conference (DAC), pages 624-629, 2006.

[24] A. Sadeghi, I. Visconti, and C. Wachsmann. Anonymizer-enabled security and privacy for RFID. In International Conference on Cryptology and Network Security (CANS), pages 134-153, 2009.

[25] M. Tehranipoor and F. Koushanfar. A survey of hardware Trojan taxonomy and detection. IEEE Design Test of Computers, 27(1):10-25, 2010.

[26] M. Tehranipoor, H. Salmani, X. Zhang, X. Wang, R. Karri, J. Rajendran, and K. Rosenfeld. Trustworthy hardware: Trojan detection and design-for-trust challenges. IEEE Computer Magazine, 44(7):66-74, 2011.

[27] A. Vahdatpour, S. Meguerdichian, and M. Potkonjak. A gate level sensor network for integrated circuits temperature monitoring. In IEEE Sensors, pages 652-655, 2010.

[28] S. Wei, K. Li, F. Koushanfar, and M. Potkonjak. Provably complete hardware trojan detection using test point insertion. In IEEE/ACM International Conference on Computer-Aided Design (ICCAD), pages 569-576, 2012.
[29] S. Wei, S. Meguerdichian, and M. Potkonjak. Gate-level characterization: Foundations and hardware security applications. In Design Automation Conference (DAC), pages 222-227, 2010.

[30] S. Wei, S. Meguerdichian, and M. Potkonjak. Malicious circuitry detection using thermal conditioning. IEEE Transactions on Information Forensics and Security, 6(3):1136-1145, 2011.

[31] S. Wei and M. Potkonjak. Scalable segmentation-based malicious circuitry detection and diagnosis. In IEEE/ACM International Conference on Computer-Aided Design (ICCAD), pages 483-486, 2010.

[32] S. Wei and M. Potkonjak. Scalable consistency-based hardware Trojan detection and diagnosis. In International Conference on Network and System Security (NSS), pages 176-183, 2011.

[33] S. Wei and M. Potkonjak. Hardware trojan horse benchmark via optimal creation and placement of malicious circuitry. In Design Automation Conference (DAC), pages 90-95, 2012.

[34] S. Wei and M. Potkonjak. Scalable hardware Trojan diagnosis. IEEE Transactions on Very Large Scale Integration (VLSI) Systems, 20(6):1049-1057, 2012.

[35] S. Wei and M. Potkonjak. Wireless security techniques for coordinated manufacturing and on-line hardware trojan detection. In ACM conference on Security and Privacy in Wireless and Mobile Networks (WiSec), pages 161-172, 2012.

[36] H. Xu, R. Vemuri, and W. Jone. Temporal and spatial idleness exploitation for optimal-grained leakage control. In IEEE/ACM International Conference on Computer-Aided Design (ICCAD), pages 468-473, 2009.

[37] L. Yuan and G. Qu. A combined gate replacement and input vector control approach for leakage current reduction. IEEE Transactions on Very Large Scale Integration (VLSI) Systems, 14(2):173-182, 2006.

[38] L. Zhang, N. Howard, V. Gumaste, A. Poddar, and L. Nguyen. Thermal characterization of stacked-die packages. In Semiconductor Thermal Measurement and Management Symposium, pages 55-63, 2004.

[39] D. Ziener and J. Teich. Power signature watermarking of IP cores for FPGAs. Journal of Signal Processing Systems, 51(1):123-136, 2008. 